\section{Microbiota supports air attack}

A large body of work has helped to define the immunological effects of tissue-associated microbiota, particularly in the intestine, but relatively little is known concerning the lung microbiota. Yang et al. show that dysregulation of the lung microbiota in a mouse model drives pulmonary fibrosis by inducing the upregulation of IL-17B. They propose that dysregulation of the pulmonary microbiota could lie at the heart of idiopathic pulmonary fibrosis (IPF), a severe lung disease of unknown aetiology.

Historically, the lower respiratory tract was considered to be sterile. However, recent studies identified a lung microbiome and there are reports that this is altered in patients with IPF. The authors used a mouse model of bleomycin-induced fibrosis to explore the effects of the microbiota in fibrotic lung disease. Bleomycin treatment induced $\sim 50 \%$ mortality in wild-type mice and this was associated with peribronchial immune cell infiltration and pulmonary fibrosis. Antibiotic-mediated depletion of lung bacteria led to reduced immune cell infiltration and fibrosis in response to bleomycin and protected against death. By contrast, depletion of fungi had no effect on bleomycin-induced pathology. Oral delivery of antibiotics to restrict microbiota depletion to the gut also did not protect mice in the bleomycin model. Therefore, the lung-resident microbiota seems to be a key driver of fibrotic disease in this

Antibiotic treatment before bleomycin administration was associated with reduced expression of IL-17 family cytokines, which have previously been linked to lung fibrosis. Deficiency of IL-17A, IL-17B or IL-17E alleviated bleomycin-induced fibrosis but these protective effects were lost if mice were treated with antibiotics targeting the lung microbiota. Therefore, induction of IL-17 family members by the lung microbiota is crucial for bleomycin-induced fibrotic disease.

As IL-17B has been less well-studied in the context of fibrosis, the authors focused on this cytokine. They used an adenoviral system to express IL-17B in lungs and found that this induced the expression of pro-inflammatory and pro-fibrotic genes. Further experiments IL-17RB-IL-17RA heterodimeric receptor complex and that IL-17RB expression on stromal cells and epithelial cells, but not on bone marrow-derived cells, was necessary for bleomycin-induced lung fibrosis. Stimulation of primary mouse type II epithelial cells or cells from a human lung epithelial cell line with IL-17B induced expression of pro-inflammatory cytokines, and IL-17B was shown to synergize with tumour necrosis factor mouse model. showed that IL-17B signals through an

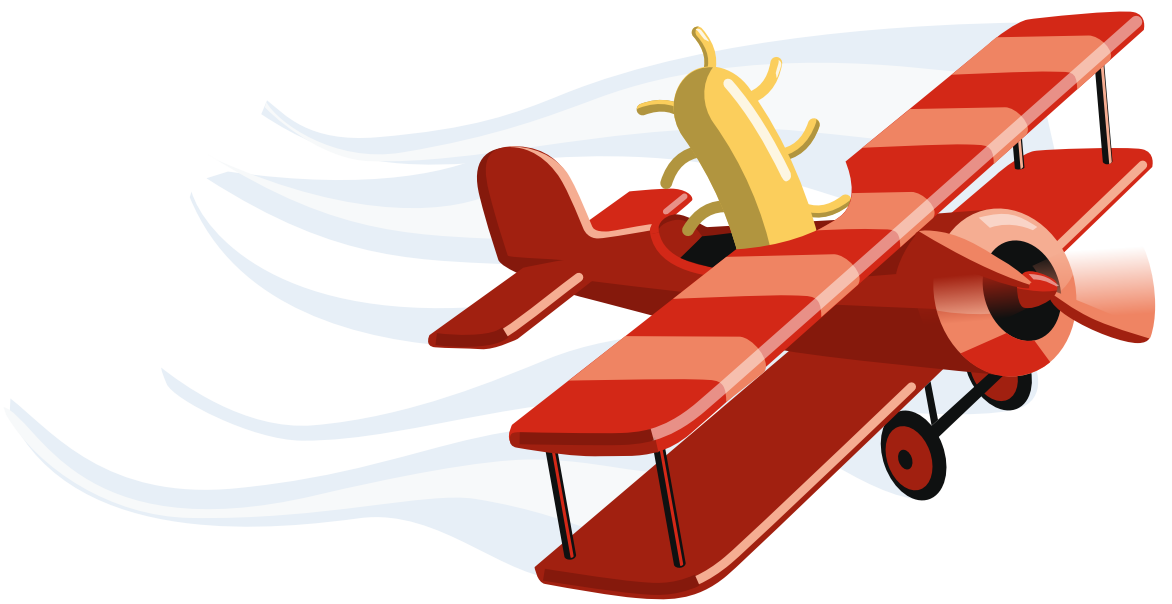

(TNF) to further promote expression of pro-inflammatory and pro-fibrotic genes in lung epithelial cells. Accordingly, in the lungs of bleomycin-treated IL-17B-deficient mice, there was decreased expression of pro-inflammatory and pro-fibrotic genes associated with $\mathrm{T}$ helper $17\left(\mathrm{~T}_{\mathrm{H}} 17\right)$ cell-type immune responses, as well as an overall reduction in neutrophils and $\mathrm{T}_{\mathrm{H}} 17$ cells.

The authors next examined the microbiota components linked to fibrosis. They intranasally inoculated microbiota-depleted mice with different bacterial species before bleomycin challenge and identified three Bacteroidetes species - Bacteroides ovatus, Bacteroides stercoris and Prevotella melaninogenica - that markedly increased lung fibrosis and mouse mortality. All of these species were expanded in the lungs of wild-type mice following bleomycin treatment; the authors linked this to an increase in host metabolites that support bacterial growth in the bleomycin-damaged lung. The three Bacteroidetes species did not induce expression of IL-17 family cytokines in the lungs of resting mice but were found to induce IL-17B and IL-17A following bleomycin treatment.

Interestingly, the authors found that outer membrane vesicles (OMVs) produced by $B$. stercoris and $P$. melaninogenica induced expression of IL-17B and IL-17A in the lungs. These OMVs upregulated IL-17B in macrophages in a process dependent on Toll-like receptor 4 (TLR4), TLR2 and MYD88, and IL-17B induction by OMVs was reduced if lipopolysaccharide was neutralized.

Therefore, bleomycin-induced lung damage can support the expansion of Bacteriodetes species that promote fibrotic responses by producing OMVs that induce IL-17B production in macrophages. Similar dysregulation of the lung microbiota may contribute to pulmonary fibrosis in humans, and the authors suggest that antibiotics or targeting IL-17B could be useful in treating IPF and other fibrotic diseases.

Yvonne Bordon, Senior Editor, Nature Reviews Immunology This article is modified from the original in Nat. Rev. Immunol. (https://doi.org/10.1038/s41577-019-0149-0). 